\title{
Cardiac-Restricted Ankyrin-Repeated Protein Is Differentially Induced in Duchenne and Congenital Muscular Dystrophy
}

\author{
Chisato Nakada, Yoshiyuki Tsukamoto, Akira Oka, Ikuya Nonaka, Shin-ichi Takeda, \\ Kenzo Sato, Shigeo Mori, Hisao Ito, and Masatsugu Moriyama
}

Division of Molecular Biology (CN, YT, KS, MM), Department of Molecular and Cellular Biology, Division of Child Neurology (AO), Institute of Neurological Science, Division of Molecular Medicine and Therapeutics (ST), Division of Organ Pathology (HI), Department of Microbiology and Pathology, Faculty of Medicine, Tottori University, Yonago, and National Center Hospital for Mental, Nervous and Muscular Disorders, National Center of Neurology and Psychiatry (IN), Tokyo, and Department of Pathology (SM), Institute of Medical Science, University of Tokyo, Tokyo, Japan

SUMMARY: Cardiac ankyrin-repeated protein (CARP) has been shown to associate with a transcription factor, YB-1, that may activate expression of the ventricular myosin light chain-2 gene during cardiogenesis. CARP is induced in the adult hypertrophic heart subjected to pressure overload, suggesting that CARP may play important functional roles in both embryonic and adult hearts. Although CARP expression was initially believed to be restricted to the heart, we found recently that CARP is induced strongly in human fetal skeletal muscle and in experimentally denervated skeletal muscle, leading us to speculate that CARP may also play important roles in skeletal muscle. In the present study, we found that in rats initially damaged by a single injection of bupivacaine, CARP expression was induced strongly in regenerating muscles with a peak 3 days after the injection, followed by down-regulation to undetectable levels after 28 days. Although CARP was coexpressed with embryonic myosin heavy chain $(\mathrm{MHC})$ in regenerating myofibers, CARP expression persisted even after down-regulation of embryonic MHC expression, whereas it began to decrease before the onset of slow or fast MHC expression, suggesting that CARP is expressed at a specific differentiation stage during muscle regeneration. We analyzed the expression of CARP in muscle biopsy specimens from 14 patients with muscular dystrophy (MD) and detected high expression of CARP in 13 of the 14 cases. CARP-positive myofibers were detected more often in congenital muscular dystrophy (CMD) than in Duchenne muscular dystrophy (DMD). We found that CARP was expressed exclusively, and at a high level, in small regenerating myofibers that express embryonic MHC in DMD, which suggested that CARP could be used as a marker of muscle regeneration in DMD. On the other hand, in CMD, expression of CARP was not limited to regenerating fibers, being detectable in myofibers expressing embryonic MHC and those expressing mature-type MHC. These findings suggest that the differentiation stage of CARP-positive myofibers in DMD and CMD may differ. (Lab Invest 2003, 83:711-719).

C ardiac ankyrin-repeated protein (CARP) was identified recently as a protein that interacts with a transcription factor, YB-1, that may target the ventricular myosin light chain-2 (MLC-2v) gene in neonatal rat heart (Zou et al, 1997). During cardiogenesis, MLC-2v protein has been shown to play important roles in the maintenance of cardiac contractility and ventricular chamber morphogenesis (Nguyen-Tran et al, 1999). Therefore, CARP is hypothesized to participate in cardiogenesis via interaction with $\mathrm{YB}-1$, pos-

DOI: 10.1097/01.LAB.0000067484.35298.1A

Received January 31, 2003.

Supported by a grant-in-aid from the Ministry of Education of Japan (Grant 12877032).

Address reprint requests to: Dr. M. Moriyama, Division of Molecular Biology, Department of Molecular and Cellular Biology, School of Life Science, Faculty of Medicine, Tottori University, 86 Nishimachi, Yonago City, Tottori 6838503, Japan.E-mail:moriyama@grape.med.tottori-u.ac.jp sibly regulating expression of the MLC-2v gene (Nguyen-Tran et al, 1999). Furthermore, it has been reported that CARP expression in the heart is upregulated by pressure overload (Aihara et al, 2000; Kuo et al, 1999). On the basis of this finding, CARP has been proposed as a potential genetic marker of cardiac hypertrophy and may have specific functional roles in the hypertrophic heart (Aihara et al, 2000; Kuo et al, 1999).

Although CARP expression has been thought to be restricted to heart and barely detectable in skeletal muscle (Jeyaseelan et al, 1997; Zou et al, 1997), we found recently that CARP is expressed in fetal skeletal muscle (Ishiguro et al, 2002), suggesting that CARP may have important functional roles in skeletal as well as cardiac muscle. Moreover, we and other investigators have also found that the expression of CARP is up-regulated transiently in denervated skeletal muscle (Baumeister et al, 1997; Tsukamoto et al, 2002). These 
findings led us to hypothesize that CARP may also be induced in skeletal muscle under specific conditions.

In this study, we first tested the regenerating muscles of rats initially damaged by a single injection of bupivacaine for expression of CARP. CARP expression was up-regulated and peaked 3 days after the bupivacaine injection, followed by down-regulation to undetectable levels 28 days after the injection. These findings suggest that CARP is induced transiently in a specific stage of differentiation in regenerating skeletal muscle.

Muscular dystrophy (MD) is an inherited muscle disease characterized pathologically by muscle necrosis, regeneration of myofibers, and interstitial fibrosis (Heffner and Schochet, 1996; Hoffman, 2001; Kaplan et al, 2001; Nonaka, 1999; Tawil and Griggs, 2001; Voit, 2001). We speculated that CARP could be induced in skeletal muscle of MD patients. To confirm this possibility, we immunohistochemically analyzed skeletal muscle from 14 patients with MD. We found that CARP was expressed strongly in regenerating myofibers of patients with Duchenne muscular dystrophy (DMD) and that most CARP-positive myofibers coexpressed embryonic myosin heavy chain (MHC), suggesting that CARP could be used as a marker of regeneration in DMD. On the other hand, in congenital muscular dystrophies (CMDs), CARP-positive myofibers were found to coexpress either embryonic or mature-type MHCs, indicating that the expression patterns of CARP, and thus the differentiation stage of CARP-positive myofibers, may differ between DMD and CMD. Furthermore, because CARP-positivity rates were significantly higher in severe cases, CARP expression may be correlated with the severity of muscle damage in MDs.

\section{Results}

\section{CARP Is Induced in Regenerating Fibers}

To determine whether CARP could be induced in regenerating myofibers, we prepared regenerating muscle by injecting bupivacaine, which is known to induce muscle necrosis (Benoit and Belt, 1970), into gastrocnemius muscles of rats and analyzed them for the expression of CARP using Western blotting. As shown in Figure 1, CARP was undetectable in noninjected muscle (Day 0), whereas it was induced on the day after injection of bupivacaine (Day 1). The protein

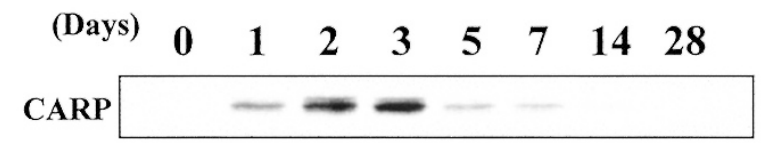

\section{Figure 1.}

Western blot analysis of rat regenerating muscles for the expression of cardiac ankyrin-repeated protein (CARP). Bupivacaine-injected rat gastrocnemius muscles were excised surgically at 1, 2, 3, 5, 7, 14, and 28 days after the injection. Proteins were extracted from the whole gastrocnemius muscles, and $40 \mu \mathrm{g}$ of each lysate was subjected to Western blot analysis with $\alpha$-mCARP(N) $\mathrm{Ab}$ (anti-mCARP antibody). Noninjected muscles were used as controls (Day $0)$. levels were up-regulated markedly, peaked 3 days after injection, and subsequently were downregulated gradually. Fourteen days after the injection, CARP was detected at only a trace level and was undetectable 28 days after injection (Fig. 1). In control rats that had been injected with saline instead of bupivacaine, CARP was undetectable throughout the experimental period (data not shown).

To confirm these observations, we further analyzed the expression of CARP using immunohistochemistry. Consistent with the Western blotting data, we found that many small mononucleated myoblast-like cells expressing CARP were distributed diffusely both inside and outside the necrotic myofibers (Fig. 2, C and D). In noninjected muscle, however, CARP-positive cells were undetectable (Fig. 2, A and B). At Day 3 after injection, small myofibers with centrally placed nuclei expressed CARP at a high level and were distributed diffusely in areas where bupivacaine-mediated necrosis had occurred (Fig. 2, E and F). Interestingly, among CARP-positive myofibers, CARP was distributed in the cytoplasm. However, in some myofibers, the nucleus and the cytoplasm were found to be immunostained positively (Fig. 2, L and M). Inspection of a longitudinal section showed that CARP was distributed diffusely throughout the cytoplasm (Fig. 2M). At Day 5 after injection, the expression levels of CARP in

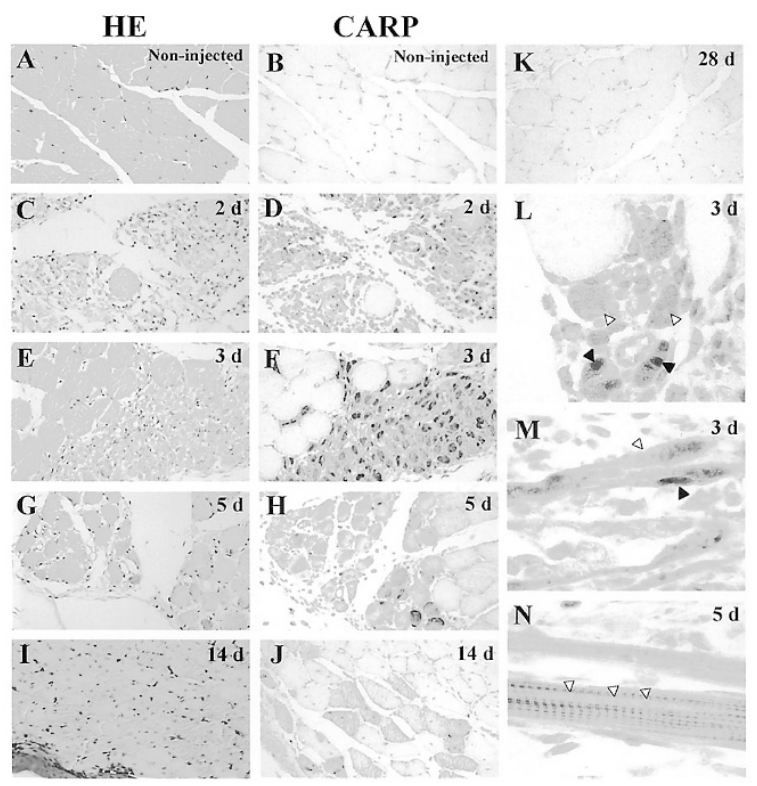

Figure 2.

Immunohistochemistry of rat regenerating muscle fibers for CARP. After the injection of bupivacaine into gastrocnemius muscles of rats, the regenerating gastrocnemius muscles were resected at 2 days $(C$ and $D), 3$ days $(E, F, L$, and $M), 5$ days ( $G, H$, and $N$ ), 14 days (I and $J)$, and 28 days (K) after the injection, and then subjected to immunohistochemistry with $\alpha$-mCARP(N)Ab (D, F, H, J, $\mathrm{K}, \mathrm{L}, \mathrm{M}$, and $\mathrm{N}$ ). Morphology was analyzed further with hematoxylin and eosin (HE) staining ( $C, E, G$, and I). The noninjected muscle was analyzed in a similar way (A and B). Original magnification, $\times 100$ (A to K). L, Immunohistochemistry of bupivacaine-injected gastrocnemius muscle 3 days after injection with $\alpha-\operatorname{mCARP}(\mathrm{N}) \mathrm{Ab}$. M and $\mathrm{N}$, Longitudinal views of bupivacaine-injected gastrocnemius muscle at 3 days (M) and at 5 days (N) after injection. CARP-expressing fibers with positive nuclear staining (solid arrowheads in $\mathrm{L}$ and $\mathrm{M}$ ) and those with negative nuclear staining (open arrowheads in $\mathrm{L}, \mathrm{M}$, and N) were admixed. Original magnification, $\times 400$ (L to N). 
the regenerating myofibers were lower, although the myofibers were still growing larger (Fig. 2, $G$ and $H$ ). Interestingly, at Day 5 after injection, observation of longitudinal sections revealed that CARP was distributed in a striated pattern in a large proportion of CARP-positive myofibers (Fig. 2N). However, in the other small proportion of CARP-positive myofibers, CARP was distributed diffusely in the cytoplasm (Fig. 3b, E1), similarly to the myofibers at Day 3 after injection, suggesting that the intracellular distribution of CARP may change during myogenic differentiation. Thereafter, down-regulation of CARP in each CARPexpressing myofiber continued (Fig. 2, I and J), and CARP was undetectable at Day 28 after injection (Fig. $2 \mathrm{~K})$. Additionally, we noticed that at Day 5 after injection, almost all of the fibers with centrally placed nuclei expressed CARP (Fig. 2, G and H), whereas at Day 14 after injection, CARP-negative fibers with centrally placed nuclei were detectable (Fig. 2, I and J). These findings suggest that CARP is induced in regenerating muscle and down-regulated to undetect- able levels before the maturation of myofibers is completed.

\section{Regenerating Muscle Fibers Coexpress Embryonic MHC and CARP}

To determine the stage at which CARP-positive myofibers differentiate in regenerating muscle, coexpression of myogenin (a marker for the earliest state of differentiation), embryonic MHC (a marker for the next earliest state of differentiation), and slow MHC (a marker for the terminal state of differentiation) was analyzed by double-labeled immunohistochemistry using $\alpha$-mCARP(N) Ab (anti-mCARP antibody) together with $\alpha$-myogenin Ab, $\alpha$-embryonic $\mathrm{MHC} \mathrm{Ab}$, or $\alpha$-slow MHC Ab. As shown in Figure $3 a$ (A1 to A3), 3 days after the injection, most of the myogenin-positive regenerating myofibers were found to coexpress CARP. Thus, CARP may be induced at an early stage of regeneration. Embryonic MHC, which is expressed transiently at the early stage of muscle development a

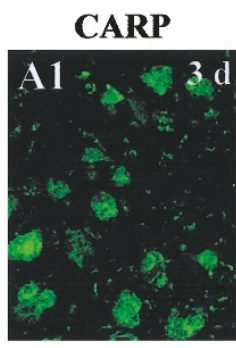

CARP

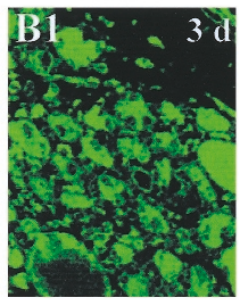

CARP

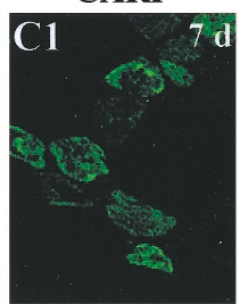

\section{Myogenin}

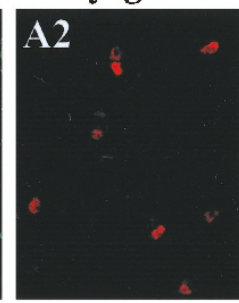

eMHC

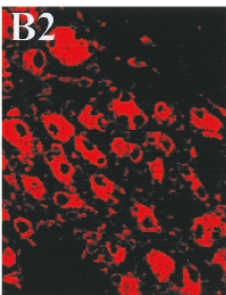

slow MHC

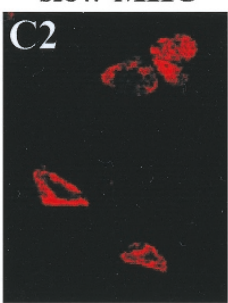

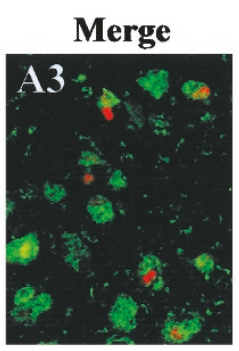

Merge

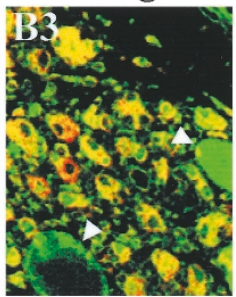

Merge

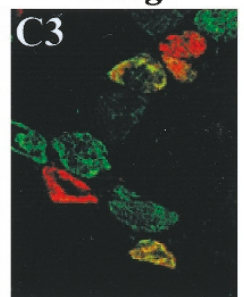

b
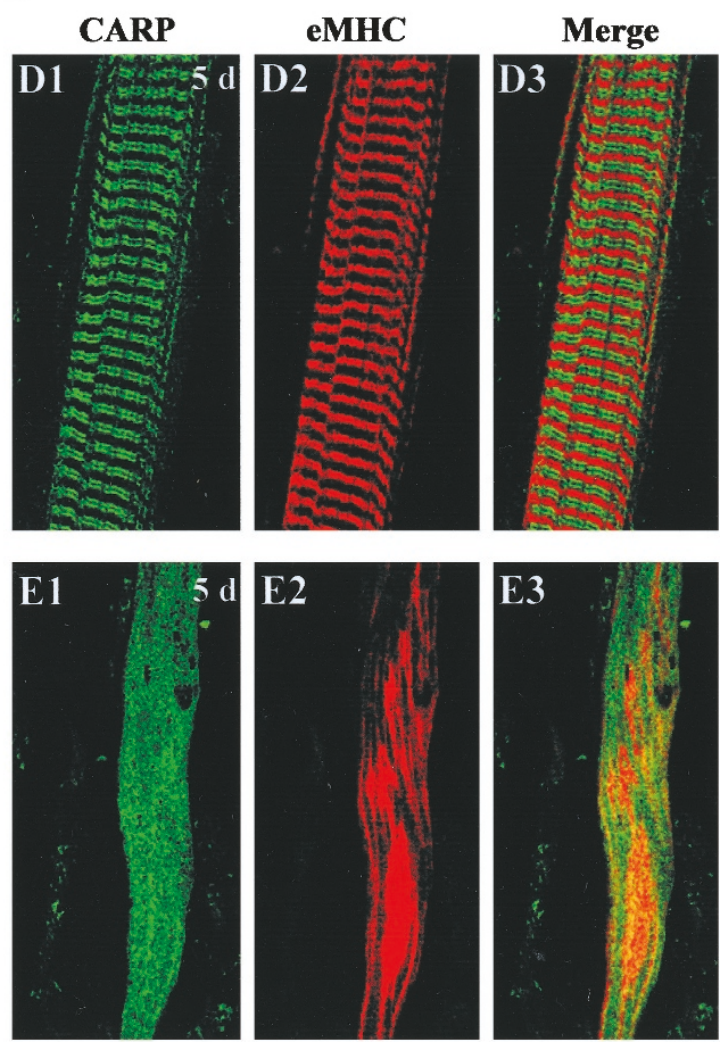

Figure 3.

Double-labeled immunohistochemistry of rat regenerating muscles. a, Coexpression of CARP with myogenin, embryonic, or slow myosin heavy chain (MHC) in rat regenerating muscle. Double-labeled immunohistochemistry was performed on the deparaffinized tissue sections of bupivacaine-injected regenerating muscles of rats at 3 days after injection with $\alpha$-mCARP(N) Ab and $\alpha$-myogenin Ab (a, A1 to A3), at 3 days after injection with $\alpha$-mCARP(N) Ab and $\alpha$-embryonic MHC Ab (a, B1 to B3), and at 7 days after injection with $\alpha$-mCARP(N) Ab and $\alpha$-slow MHC Ab (a, C1 to C3). A mixture of Alexa Fluor 546-conjugated goat anti-mouse antibody and Alexa Fluor 488-conjugated goat anti-rabbit antibody was used as secondary antibodies. CARP is shown as green signals and embryonic MHC and myogenin are shown as red signals. Merged signals are detected as yellow signals. Arrowheads in B3 show the CARP-positive myofibers that do not express embryonic MHC. Original magnification, $\times 200$. b, Double-labeled immunohistochemistry of longitudinal sections of rat regenerating muscles at 5 days after bupivacaine injection. In CARP-positive myofibers in which CARP was distributed in a striated pattern, CARP and embryonic MHC were distributed in an antiparallel manner (D1 to D3). In the other CARP-positive myofibers in which CARP was diffusely distributed, embryonic MHC was distributed in a longitudinally oriented filamentous pattern and was not colocalized with CARP (E1 to E3). Original magnification, $\times 600$. eMHC $=$ embryonic MHC. 
(LaFramboise et al, 1990; Schiaffino et al, 1986), was detectable in small regenerating fibers 3 days after bupivacaine injection (Fig. 3a, B1 to B3). Furthermore, almost all of the embryonic $\mathrm{MHC}$-positive regenerating fibers coexpressed CARP and were distributed diffusely throughout the areas where bupivacaineinduced necrosis had occurred (Fig. 3a, B1 to B3). Large CARP-positive but embryonic MHC-negative myofibers were found scattered at the periphery of the mass of small regenerating fibers (Fig. 3a, B3, arrowhead). At Day 7 after bupivacaine injection, the population of embryonic MHC-positive regenerating fibers was smaller, although the population of CARPpositive fibers was unchanged (data not shown). Consequently, CARP-positive but embryonic MHCnegative regenerating fibers were proportionately more abundant.

On the other hand, slow MHC, which is expressed in mature myofibers, was undetectable at Day 5 after injection, but markedly induced in some CARP-positive regenerating fibers at Day 7 after injection (Fig. $3 a, \mathrm{C}_{1}$ to $\mathrm{C} 3$ ). These results indicate that, during the regeneration process, CARP was induced strongly and peaked in the early stage of differentiation and then was down-regulated to undetectable levels, accompanied by up-regulation of slow or fast MHC. When we analyzed longitudinal sections, we noticed that the intracellular distribution of CARP changed during the differentiation of CARP-positive regenerating myofibers. At Day 5 after injection, in a large proportion of CARPpositive myofibers, CARP was distributed in a striated pattern (Fig. 2, M and N). Double immunohistochemistry with $\alpha$-mCARP Ab and $\alpha$-embryonic $\mathrm{MHC} A \mathrm{~b}$, which is localized almost exclusively to the sarcomeric M-band, revealed that positive signals for CARP and those for embryonic MHC were not colocalized but rather arranged in an antiparallel manner (Fig. 3b, D1 to D3). These findings suggested that CARP may be localized in the sarcomeric I-Z-I areas. In a small proportion of CARPpositive myofibers, CARP was distributed diffusely throughout the cytoplasm (Fig. 3b, E1). A similar diffuse expression pattern was also seen in CARPpositive myofibers at Day 3 after injection (Fig. 2M).

\section{CARP Is Expressed Differentially in Muscular Dystrophies}

To evaluate the expression of CARP in MDs, we analyzed skeletal muscle samples taken from $14 \mathrm{MD}$ patients using immunohistochemistry. In normal muscles, consistent with our previous study (Ishiguro et al, 2002), CARP was barely detectable (Fig. 4B), whereas in MDs, the fibers were strongly positive for CARP (Fig. 4D, F, H, J). As shown in Table 1 , in 13 of 14 cases of MD, CARP-positive fibers were detected, and this was confirmed by quantitative analysis (Fig. 5). The positivity rates for CARPexpressing fibers were apparently higher in CMDs than in the other subtypes (Fig. 5, Table 1). In cross-sections from patients with DMD and limbgirdle MD, CARP-positive myofibers tended to be
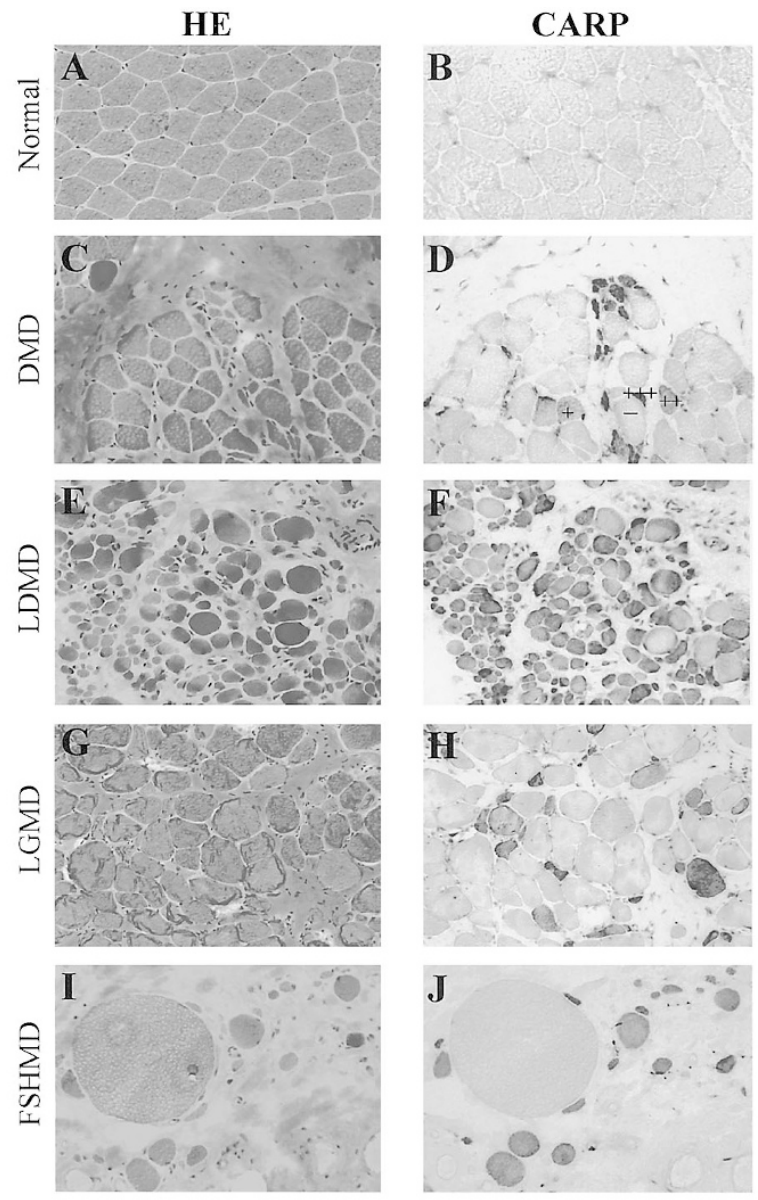

Figure 4.

Immunohistochemistry of biopsy specimens from muscular dystrophy (MD) patients for expression of CARP. Frozen tissue sections from MD patients [Duchenne muscular dystrophy (DMD) (C and D), laminin- $\alpha 2$ deficient muscular dystrophy (LDMD) ( $E$ and $F$ ), limb-girdle muscular dystrophy (LGMD) (G and H), and facioscapulohumeral muscular dystrophy (FSHMD) (I and J)] and a sample of normal control muscle (A and B) were analyzed using immunohistochemistry with $\alpha$-hCARP(N) (B, D, F, H, and $\mathrm{J}$ ) and $\mathrm{HE}$ staining $(A, C, E, G$, and I) (original magnification, $\times 100$ ). CARP-positive fibers were not detected in normal muscle $(B)$, whereas in MDs, small CARP-positive fibers were scattered around large CARPnegative fibers $(D, H$, and $J)$. Strongly immunoreactive $(+++)$, moderately immunoreactive $(++)$, weakly immunoreactive $(+)$, and nonimmunoreactive $(-)$ muscle fibers are illustrated in $D$.

small, round, and distributed in groups around large fibers (Fig. 4, D and H). Furthermore, we noticed that fibers with centrally placed nuclei tended to express CARP (data not shown). Because these phenotypes found in CARP-positive fibers are similar to those seen in regenerating myofibers (Carpenter, 2001; Nonaka, 1999; Sewry and Dubowitz, 2001), we hypothesized that CARP may be expressed preferentially in regenerating muscles of DMD patients. On the other hand, in Fukuyama congenital muscular dystrophy (FCMD) and laminin- $\alpha 2$-deficient MD, a large proportion of myofibers are likely to express CARP regardless of the fiber size and shape, and CARP-positive fibers tended to be distributed diffusely (Figs. 4F and 5). A muscle specimen from a patient with facioscapulohumeral MD exhibited a striking dystrophic change, and most of the myofi- 
Table 1. Expression of ARPP and CARP protein in muscular dystrophies

\begin{tabular}{lc}
\hline Histologic examination & CARP \\
\hline 1. DMD & + \\
2. DMD & ++ \\
3. DMD & ++ \\
4. DMD & ++ \\
5. DMD & ++ \\
6. DMD & ++ \\
7. BMD & - \\
8. FCMD & +++ \\
9. FCMD & ++ \\
10. FCMD & +++ \\
11. LDMD & +++ \\
12. LGMD & + \\
13. LGMD & ++ \\
14. FSHMD & +++ \\
\hline
\end{tabular}

BMD, Becker muscular dystrophy; LDMD, laminin- $\alpha 2$-deficient muscular dystrophy; LGMD, limb-girdle muscular dystrophy; FSHMD, facioscapulohumeral muscular dystrophy. Immunoreactivities were classified based on the population of the positive fiber:,$+++>50 \%$;,$++ 10-50 \%$;,$+ 1-10$; - , $0-1 \%$.

bers were strongly positive for CARP. Interestingly, the extremely hypertrophic myofibers were negative for CARP (Fig. 4J).

\section{Regenerating Myofibers in DMDs Express CARP}

To confirm that CARP is expressed in regenerating myofibers of DMD patients, we analyzed DMD biopsy samples by double-labeled immunohistochemistry using $\alpha$-hCARP(N) Ab (anti-hCARP antibody) together with $\alpha$-embryonic MHC Ab, $\alpha$-slow MHC Ab, or $\alpha$-fast MHC Ab. A large proportion of CARP-positive small fibers that clustered around CARP-negative myofibers were found to coexpress embryonic MHC (Fig. 6a, A1 to A3). The populations of CARP-positive fibers that coexpressed slow or fast MHC were much smaller than those that coexpressed embryonic MHC (Fig. 6a, B1 to B3, C1 to C3). Thus, the expression patterns of $\mathrm{MHC}$ isoforms were very similar to those of regenerating myofibers in that CARP-positive myofibers tended to coexpress embryonic MHC. This tendency suggests that, in DMDs, most of the CARP-positive fibers may coincide with regenerating myofibers.

\section{Difference in Expression Pattern of CARP Between DMD and CMD}

The expression patterns of CARP in CMDs, including FCMD and laminin- $\alpha 2$-deficient MD, were different from those in DMDs. As shown in Figures 4F and 5, in laminin- $\alpha 2$-deficient $\mathrm{MD}$ and FCMD, the positivity rates for CARP were significantly higher than those in DMD. Furthermore, CARP was expressed regardless of the size or shape of the myofibers in CMDs. In laminin- $\alpha 2$-deficient MD or FCMD, only some CARPpositive fibers coexpressed embryonic MHC (Fig. 6b, D1 to D3), but the other CARP-positive fibers coexpressed slow or fast MHC (Fig. 6b, E1 to E3, F1 to F3).

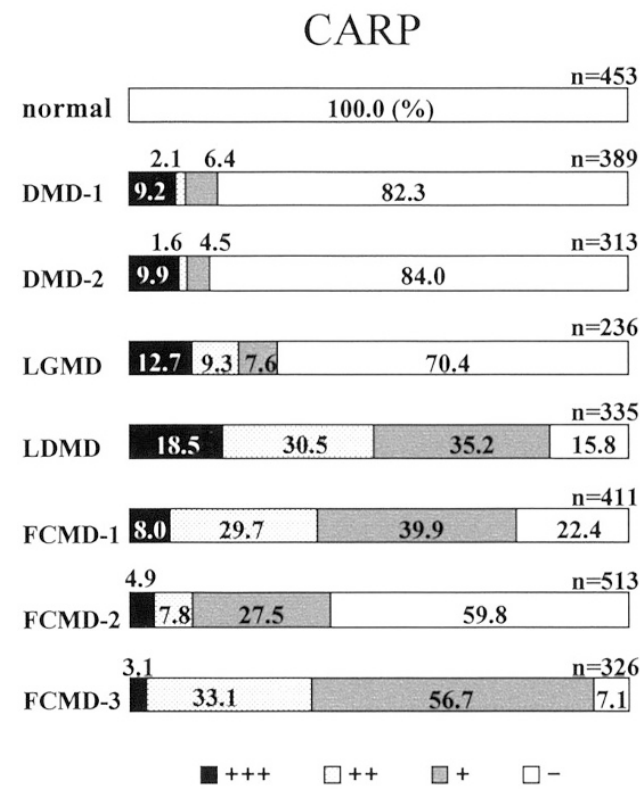

Figure 5.

Populations of CARP-positive fibers in MDs. Populations of CARP-positive myofibers were analyzed in two cases of DMDs, one case of LGMD, one case of LDMD, and three cases of Fukuyama congenital muscular dystrophy (FCMD). Immunohistochemically positive myofibers were categorized into four groups: strongly immunoreactive $(+++)$, moderately immunoreactive $(++)$, weakly immunoreactive $(+)$, and nonimmunoreactive $(-)$ muscle fibers, according to the intensity of their immunoreactivities for CARP (see Fig. 4D). In each case, 236 to 513 fibers were examined according to the above criteria, and the total number of fibers is indicated as $n$ for each case. Percentages of positive fibers are shown in the bar graphs.

These results suggest that, in CMDs including FCMD and laminin- $\alpha 2$-deficient MD, CARP expression may not be confined to regenerating myofibers.

\section{Discussion}

It has been reported that CARP is localized in the nucleus and interacts with a transcription factor, YB-1 (Zou et al, 1997). Coexpression of CARP and YB-1 has been shown to repress the transcriptional target of YB-1, MLC-2v, which is required for the maintenance of cardiac contractility and ventricular chamber morphogenesis (Nguyen-Tran et al, 1999). However, our group and another group recently reported that CARP was localized mainly in the cytoplasm as well as in the nucleus of fetal and adult heart (Bang et al, 2001; Ishiguro et al, 2002). Furthermore, we showed in this study that CARP is localized in the sarcomeric I-Z-I areas of regenerating skeletal muscle. This observation is consistent with a recent study showing that CARP is localized at sarcomeric I-bands in mouse cardiomyocytes (Bang et al, 2001). It was proposed recently that CARP may have a role in the maintenance of sarcomeric structure via interaction with a newly identified protein, myopalladin, which is localized in the I-bands of myofibers (Bang et al, 2001). Interestingly, when this interaction was perturbed by introduction of a dominant-negative form of myopalladin that binds competitively to CARP, the sarcomeric structure of the myofiber was destroyed, sug- 
a
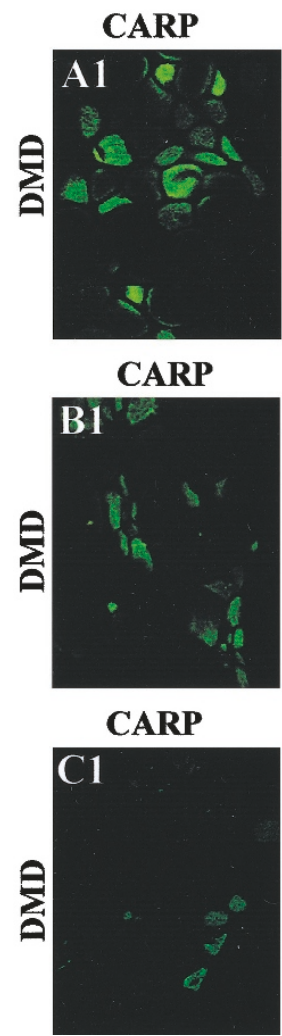

eMHC

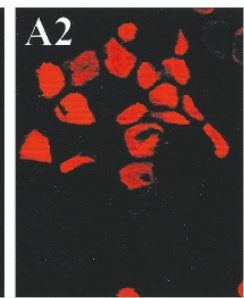

slow MHC

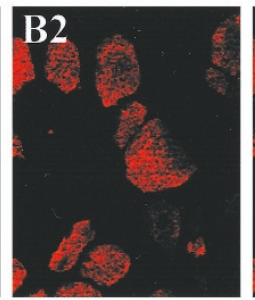

fast MHC

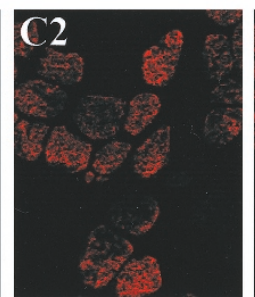

Merge

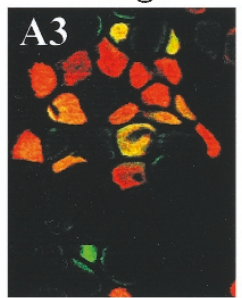

Merge

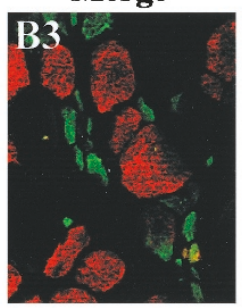

Merge

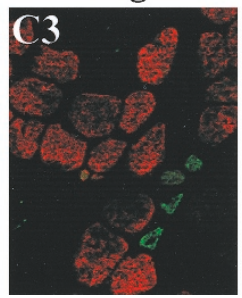

b
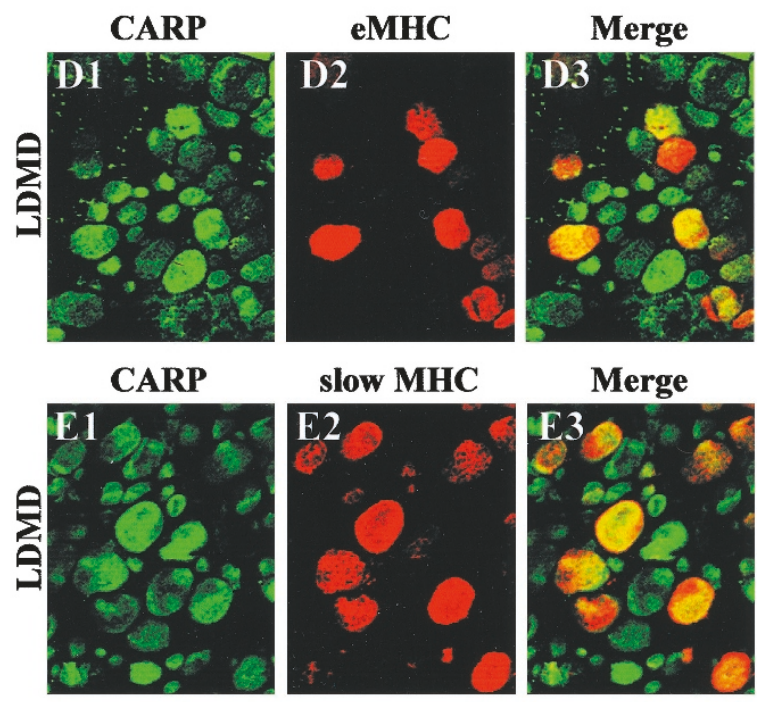

CARP
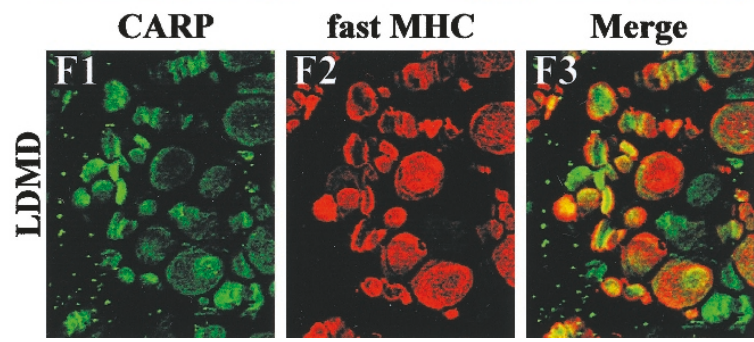

Figure 6 .

Double-labeled immunohistochemistry of human MDs. a, Coexpression of CARP with embryonic, slow, or fast MHC in MDs. Frozen tissue sections of the DMD cases were analyzed by double-labeled immunohistochemistry for the coexpression of CARP with embryonic MHC (A1 to A3), slow MHC (B1 to B3), or fast MHC (C1 to C3) using $\alpha$-hCARP(N) Ab together with $\alpha$-embryonic MHC Ab, $\alpha$-slow MHC Ab, or $\alpha$-fast MHC Ab. Original magnification, $\times 200$. b, Coexpression of CARP with embryonic, slow, or fast MHC in MDs. Frozen tissue sections of the LDMD case were analyzed by double-labeled immunohistochemistry for the coexpression of CARP with embryonic MHC (D1 to D3), slow MHC (E1 to E3), or fast MHC (F1 to F3). Original magnification, $\times 200$.

gesting that interaction of CARP with myopalladin is required to maintain sarcomeric integrity (Bang et al, 2001).

In the present study, CARP was expressed at a high level 3 days after bupivacaine injection. Thereafter, though, it was gradually down-regulated and became undetectable 28 days after the injection. Furthermore, analysis of its intracellular localization at Day 3 after injection revealed that CARP was distributed diffusely throughout the cytoplasm. However, 5 days after injection, CARP was distributed in the sarcomeric I-Z-I areas in a striated pattern. In some CARP-positive myofibers in which the sarcomere was not yet fully constructed 5 days after injection, CARP was distributed diffusely, suggesting that intracellular distribution of CARP may change during sarcomerogenesis. Fusion of myoblasts and sarcomerogenesis have been shown to occur 3 or 4 days after injection with mepivacaine or bupivacaine (Carlson and Rainin, 1985; Okland et al, 1989). Therefore, our present data and these reports lead us to speculate that the highly induced CARP in regenerating skeletal muscle may participate in sarcomerogenesis or the maintenance of sarcomeric structure during regeneration. However, we cannot rule out the possibility that CARP may also participate in transcriptional regulation via interaction with YB-1 in regenerating myofibers as well as in cardiomyocytes, because CARP was localized transiently in the nucleus during regeneration (Fig. 2L).

In DMD, CARP tends to be expressed in small myofibers that are morphologically similar to regenerating myofibers. Furthermore, CARP-positive myofibers coexpressed embryonic MHC but usually did not coexpress slow or fast MHC. These expression patterns of CARP in DMD are also very similar to those in experimentally induced regenerating muscle, which suggests that CARP may be induced during regeneration after muscle necrosis in DMD.

In CMD, in contrast to DMD, CARP was expressed in both embryonic MHC-positive regenerating myofibers and fast or slow MHC-positive mature myofibers. This trend was observed in both laminin- $\alpha 2$-deficient $M D$ and FCMD. These findings suggest that CARP expression in CMD is not limited to regenerating myofibers and that the stage of differentiation of CARP-positive myofibers in DMD and CMD may be different. We cannot explain fully the mechanisms responsible for the induction of CARP in CMD at this stage. However, the extreme vulnerability of myofibers and the presence of muscle degeneration at birth may contribute to the constitutive expression of CARP in CMD. 
Becker MD, a milder form of dystrophin-defective $\mathrm{MD}$, has a protracted clinical course, with far fewer necrotic and regenerating myofibers, and was not observed to express CARP in this study (Table 1). On the other hand, a muscle specimen from a patient with facioscapulohumeral MD who exhibited a striking dystrophic change contained numerous CARPexpressing fibers (Table 1, Fig. 4). These findings suggest that the various extents of induction of CARP in distinct subtypes of MD may depend on the severity of dystrophic changes in myofibers. If CARP really does have an important function in the preservation of sarcomeric integrity, it is hypothesized that the induced CARP in MDs may contribute to the maintenance of myofiber structure.

During the regeneration process, CARP was expressed in myofibers in the early stage of differentiation. Indeed, CARP induction in regenerating myofibers was found to precede the onset of mature-type $\mathrm{MHC}$ expression, and it lasted even after the downregulation of myogenin and embryonic $\mathrm{MHC}$, suggesting that the expression pattern of CARP during regeneration is distinct from existing markers of myogenic differentiation. Therefore, it seems possible that CARP may be used as a new marker of myogenic differentiation and that it may also be useful for monitoring the active regeneration process in DMDs. In this study, we found that CARP is constitutively expressed in myofibers of CMD, less frequently expressed in myofibers of DMD, and expressed hardly at all in normal skeletal muscle. Accordingly, constitutive expression of CARP may prove useful for the pathologic diagnosis of CMD. Further studies involving many more patients are required to address this possibility.

\section{Materials and Methods}

\section{Tissues}

Fourteen specimens of unfixed, frozen biceps muscle tissue taken from MD patients for diagnostic purposes and stored at $-70^{\circ} \mathrm{C}$ were used. The specimens included six cases of DMD, one case of Becker MD, two cases of limb-girdle MD, one case of facioscapulohumeral MD, and four cases of CMD (including three cases of FCMD and one case of laminin- $\alpha 2$-deficient $\mathrm{MD})$. The use of these tissue samples for the study was approved by the Institutional Review Board of Tottori University (Permission No. 186).

\section{Antibodies}

$\alpha$-hCARP(N) Ab and $\alpha$-mCARP(N) Ab were raised in our laboratory against the N-terminal 69 amino acids of human and mouse CARP protein, respectively. The characteristics of these Abs have been described in detail previously (Ishiguro et al, 2002; Tsukamoto et al, 2002). The following commercial mAbs were used in the immunohistochemical analyses: anti-embryonic MHC Ab (ARP, Belmont, Massachusetts), anti-slow MHC Ab (YLEM, Rome, Italy), anti-fast MHC Ab (YLEM), and anti-myogenin $\mathrm{Ab}$ (Santa Cruz, California).

\section{Animals}

Twenty-eight adult Wistar rats were purchased from Japan SLC (Shizuoka, Japan). Bupivacaine $(0.5 \mathrm{ml}$ of $0.5 \% \mathrm{w} / \mathrm{v}$ in saline solution) was injected into the gastrocnemius muscle of rats at 8 to 9 weeks of age (Benoit and Belt, 1970). Control rats were injected with saline alone. At 1, 2, 3, 5, 7, 14, and 28 days after the bupivacaine injection, the gastrocnemius muscles were resected and then subjected to immunohistochemistry and Western blotting. All experiments were performed in accordance with the guidelines of animal care and use established by Tottori University (Permission No. 01-S-91).

\section{Western blotting}

Whole gastrocnemius muscles of rats were resected and lysed in SDS-modified RIPA buffer [0.1\% (w/v) SDS, 40 mм HEPES-NaOH (pH 7.4), 1\% (v/v) Nonidet $\mathrm{P}-40,0.5 \%(\mathrm{w} / \mathrm{v})$ sodium deoxycholate, $150 \mathrm{~mm} \mathrm{NaCl}$, $1 \mathrm{~mm}$ phenylmethylsulfonyl fluoride, $10 \mathrm{~mm}$ sodium pyrophosphate, $10 \mathrm{~mm}$ sodium fluoride, $4 \mathrm{~mm}$ EDTA, and $2 \mathrm{~mm}$ sodium vanadate] on ice, for 90 minutes, then centrifuged at $15,000 \mathrm{rpm}$ at $4^{\circ} \mathrm{C}$ for 20 minutes. The resulting lysates (40- $\mu \mathrm{g}$ samples) were boiled with Laemmli's sample buffer and subjected to SDS (10\% w/v)-PAGE. The samples were transferred to a polyvinylidene difluoride membrane (Millipore, Bedford, Massachusetts), which was blocked for 1 hour in $10 \%$ $(\mathrm{w} / \mathrm{v})$ skim milk in $1 \times$ Tris-buffered saline (TBS) at room temperature (RT), and then incubated for 1 hour at $4^{\circ} \mathrm{C}$ with $1 \mu \mathrm{g} / \mathrm{ml} \alpha$-mCARP(N) Ab. The filter was washed thoroughly with $1 \times$ TBS containing $0.1 \%(\mathrm{v} / \mathrm{v})$ Tween 20 and then incubated for 1 hour at $4^{\circ} \mathrm{C}$ with a donkey anti-rabbit lgG horseradish peroxidase-linked whole antibody (Amersham-Pharmacia Biotech) and rewashed with $1 \times$ TBS containing $0.1 \%(v / v)$ Tween 20. Finally, the signals were detected with the ECL Western blotting analysis system (AmershamPharmacia Biotech) according to the manufacturer's instructions.

\section{Immunohistochemistry}

Paraffin-embedded tissue sections were prepared as follows. Rat gastrocnemius muscles were fixed with $10 \%(\mathrm{v} / \mathrm{v})$ formalin for 16 hours, dehydrated with ethanol, and then embedded in paraffin after progressive xylene washes. The tissue sections were thoroughly deparaffinized and rehydrated according to standard protocols. For antigen retrieval, the sections were immersed in $10 \mathrm{~mm}$ sodium citrate buffer ( $\mathrm{pH}$ 6.0) (latron Company, Tokyo, Japan), autoclaved at $120^{\circ} \mathrm{C}$ for 10 minutes, and cooled to RT. They were then treated with $3 \%(\mathrm{v} / \mathrm{v}) \mathrm{H}_{2} \mathrm{O}_{2}$ for 5 minutes at $\mathrm{RT}$ to inactivate endogenous peroxidase activity and blocked with $10 \%(\mathrm{v} / \mathrm{v})$ goat serum (Nichirei, Tokyo, Japan) for 30 minutes at RT.

Frozen tissue sections were prepared as follows. The biopsy samples of biceps muscle were snapfrozen in isopentane cooled in liquid nitrogen, and then tissue sections were prepared with a cryostat. 
After fixation in acetone at $-20^{\circ} \mathrm{C}$ for 1 hour, these sections were washed with $1 \times \mathrm{PBS}$, treated with $3 \%$ (v/v) $\mathrm{H}_{2} \mathrm{O}_{2}$ for 5 minutes at $\mathrm{RT}$, and blocked with $10 \%$ (v/v) goat serum (Nichirei) for 30 minutes at RT.

Subsequently, immunohistochemistry with $\alpha$-mCARP(N) or $\alpha$-hCARP(N) Ab was performed as follows. The tissue sections were incubated with the first $\mathrm{Ab}-\alpha$-hCARP(N) Ab or $\alpha$-mCARP(N) Ab-diluted to 1:2000 with diluting solution (DAKO, Carpenteria, California) for 18 hours at $4^{\circ} \mathrm{C}$. The sections were then washed with $1 \times$ PBS and incubated for 30 minutes with biotinylated goat antirabbit IgG (Nichirei). After being washed with $1 \times$ PBS, they were incubated with a solution of avidin-conjugated horseradish peroxidase (Vectastain Elite ABC kit; Vector Laboratories Inc., Burlingame, California) for 15 minutes, according to the manufacturer's recommendations, and then washed again with $1 \times$ PBS for 5 minutes. Peroxidase activity was detected with $\mathrm{H}_{2} \mathrm{O}_{2}$ /diaminobenzidine substrate solution, and the sections were counterstained with hematoxylin before dehydration and mounting.

\section{Double-Labeled Immunohistochemistry}

Paraffin-embedded tissue sections were deparaffinized and immersed in $10 \mathrm{~mm}$ sodium citrate buffer (pH 6.0) (latron Company) and autoclaved at $120^{\circ} \mathrm{C}$ for 10 minutes. Frozen tissue sections were fixed in acetone at $-20^{\circ} \mathrm{C}$ for 1 hour. Subsequently, they were blocked with $10 \%(\mathrm{v} / \mathrm{v})$ normal goat serum (Nichirei Company) for 30 minutes at RT. The sections were then incubated for 18 hours at $4^{\circ} \mathrm{C}$ with either the $\alpha-\operatorname{mCARP}(\mathrm{N}) \mathrm{Ab}$ or the $\alpha-\mathrm{hCARP}(\mathrm{N}) \mathrm{Ab}$ diluted to $1: 1000$, together with the $\alpha$-myogenin Ab diluted to $1: 500$, the $\alpha$-embryonic MHC Ab diluted to $1: 10$, the $\alpha$-slow MHC Ab diluted to 1:20, or the $\alpha$-fast MHC Ab diluted to 1:20. After being washed with $1 \times$ PBS, sections were incubated for 2 hours at RT with a mixture of Alexa Fluor 488-conjugated goat anti-rabbit antibody diluted to 1:200 and Alexa Fluor 546conjugated goat anti-mouse antibody (Molecular Probes) diluted to $1: 200$ in $1 \times$ PBS. They were then washed with $1 \times$ PBS and mounted with gel/mount (Biomeda Corporation). The mounted sections were observed with a fluorescence microscope (Eclipse E800; Nikon), and the images were processed with the MRC-1024 confocal system (Bio-Rad).

\section{References}

Aihara $\mathrm{Y}$, Kurabayashi M, Saito $\mathrm{Y}$, Ohyama $\mathrm{Y}$, Tanaka $\mathrm{T}$, Takeda S, Tomaru K, Sekiguchi K, Arai M, Nakamura T, and Nagai $R$ (2000). Cardiac ankyrin repeat protein is a novel marker of cardiac hypertrophy: Role of M-CAT element within the promoter. Hypertension 36:48-53.

Bang ML, Mudry RE, McElhinny AS, Trombitas K, Greach AJ, Yamasaki R, Sorimachi H, Granzier H, Gregorio CC, and Labeit S (2001). Myopalladin, a novel 145-kilodalton sarcomeric protein with multiple roles in Z-disc and I-band protein assemblies. J Cell Biol 153:413-427.

Baumeister A, Arber S, and Caroni P (1997). Accumulation of muscle ankyrin repeat protein transcript reveals local activation of primary myotube endcompartments during muscle morphogenesis. J Cell Biol 139:1231-1242.
Benoit PW and Belt WD (1970). Destruction and regeneration of skeletal muscle after treatment with a local anaesthetic, bupivacaine (Marcaine). J Anat 107:547-556.

Carlson BM and Rainin EA (1985). Rat extraocular muscle regeneration. Arch Ophthalmol 103:1373-1377.

Carpenter S (2001). Muscle pathology on semithin resin sections. In: Karpati G, Hilton-Jones D, and Griggs RC, editors. Disorders of voluntary muscle. Cambridge: Cambridge University Press, 283-295.

Heffner RR Jr and Schochet SS Jr (1996). Skeletal muscle. In: Damjanov I and Linder J, editors. Anderthon's pathology. St. Louis: Mosby, 2657-2690.

Hoffman EP (2001). Dystrophinopathies. In: Karpati G, HiltonJones D, and Griggs RC, editors. Disorders of voluntary muscle. Cambridge: Cambridge University Press, 385-432.

Ishiguro N, Baba T, Ishida T, Takeuchi K, Osaki M, Araki N, Okada E, Takahashi S, Saito M, Watanabe M, Nakada C, Tsukamoto Y, Sato K, Ito K, Fukayama M, Mori S, Ito H, and Moriyama M (2002). Carp, a cardiac ankyrin-repeated protein, and its new homologue, Arpp, are differentially expressed in heart, skeletal muscle, and rhabdomyosarcomas. Am J Pathol 160:1767-1778.

Jeyaseelan R, Poizat C, Baker RK, Abdishoo S, Isterabadi LB, Lyons GE, and Kedes L (1997). A novel cardiac-restricted target for doxorubicin: CARP, a nuclear modulator of gene expression in cardiac progenitor cells and cardiomyocytes. J Biol Chem 272:22800-22808.

Kaplan J-C, Beckmann JS, and Fardeau M (2001). Limb girdle muscular dystrophies. In: Karpati G, Hilton-Jones D, and Griggs RC, editors. Disorders of voluntary muscle. Cambridge: Cambridge University Press, 433-463.

Kuo H, Chen J, Ruiz-Lozano P, Zou Y, Nemer M, and Chien KR (1999). Control of segmental expression of the cardiacrestricted ankyrin repeat protein gene by distinct regulatory pathways in murine cardiogenesis. Development 126:42234234.

LaFramboise WA, Daood MJ, Guthrie RD, Butler-Browne GS, Whalen RG, and Ontell M (1990). Myosin isoforms in neonatal rat extensor digitorum longus, diaphragm, and soleus muscles. Am J Physiol 259:L116-L122.

Nguyen-Tran VTB, Chen J, Ruiz-Lozano P, and Chien KR (1999). The MLC-2 paradigm for ventricular heart chamber specification, maturation, and morphogenesis. In: Harvey RP and Rosenthal N, editors. Heart development. New York: Academic Press, 255-272.

Nonaka I (1999). Muscle pathology for physicians (in Japanese). Tokyo: Nihon-iji-shinposha, 2-75.

Okland S, Komorowski TE, and Carlson BM (1989). Ultrastructure of mepivacaine-induced damage and regeneration of rat extraocular muscle. Invest Ophthalmol Vis Sci 30:16431651.

Schiaffino S, Gorza L, Sartore S, Saggin L, and Carli M (1986). Embryonic myosin heavy chain as a differentiation marker of developing human skeletal muscle and rhabdomyosarcoma. Exp Cell Res 163:211-220.

Sewry CA and Dubowitz V (2001). Histochemistry and immunocytochemistry of muscle in health and disease. In: Karpati G, Hilton-Jones D, and Griggs RC, editors. Disorders of voluntary muscle. Cambridge, Cambridge University Press, 251-282. 
Tawil R and Griggs RC (2001). Facioscapulohumeral dystrophy. In: Karpati G, Hilton-Jones D, and Griggs RC, editors. Disorders of voluntary muscle. Cambridge, Cambridge University Press, 464-470.

Tsukamoto $\mathrm{Y}$, Senda T, Nakano T, Nakada C, Hida T, Ishiguro N, Kondo G, Baba T, Sato K, Osaki M, Mori S, Ito H, and Moriyama M (2002). Arpp, a new homolog of Carp, is preferentially expressed in type 1 skeletal muscle fibers and is markedly induced by denervation. Lab Invest 82:645-655.
Voit T (2001). Congenital muscular dystrophies. In: Karpati G, Hilton-Jones D, and Griggs RC, editors. Disorders of voluntary muscle. Cambridge: Cambridge University Press, 503524.

Zou Y, Evans S, Chen J, Kuo H-C, Harvey RP, and Chien KR (1997). CARP, a cardiac ankyrin repeat protein, is downstream in the Nkx2-5 homeobox gene pathway. Development 124:793-804. 